\title{
Use of EHRs in a Tertiary Hospital During COVID-19 Pandemic: A Multi-Purpose Approach Based on Standards
}

\author{
Miguel PEDRERA ${ }^{\mathrm{a}, \mathrm{b}, 1}$, Noelia GARCIA ${ }^{\mathrm{a}}$, Alvar BLANCO $^{\mathrm{a}}$, Ana Isabel TERRIZA ${ }^{\mathrm{a}}$, \\ Jaime CRUZ ${ }^{\text {a }}$, Elena Ana LOPEZ ${ }^{\mathrm{a}}$, Fernando CALVO ${ }^{\mathrm{a}}$, María Jesús JIMENEZ ${ }^{\mathrm{a}}$, \\ Patricia GONZALEZ ${ }^{a}$, Víctor QUIROS ${ }^{a}$, Juan Luis CRUZ ${ }^{a}$, José Luis BERNAL ${ }^{a}$ and \\ Pablo SERRANO \\ ${ }^{\mathrm{a}}$ Hospital Universitario 12 de Octubre, Madrid, Spain \\ ${ }^{\mathrm{b}}$ ETSI Telecomunicación, Universidad Politécnica de Madrid, Madrid, Spain
}

\begin{abstract}
This work aims to describe how EHRs have been used to meet the needs of healthcare providers and researchers in a 1,300-beds tertiary Hospital during COVID-19 pandemic. For this purpose, essential clinical concepts were identified and standardized with LOINC and SNOMED CT. After that, these concepts were implemented in EHR systems and based on them, data tools, such as clinical alerts, dynamic patient lists and a clinical follow-up dashboard, were developed for healthcare support. In addition, these data were incorporated into standardized repositories and COVID-19 databases to improve clinical research on this new disease. In conclusion, standardized EHRs allowed implementation of useful multipurpose data resources in a major Hospital in the course of the pandemic.
\end{abstract}

Keywords. Electronic Health Records, COVID-19, Information model, Standards, Semantic interoperability, Real World Data. SNOMED CT, LOINC, i2b2, RedCap.

\section{Introduction}

COVID-19 has been the major health challenge in recent decades, being declared a state of pandemic on March 11, 2020 by WHO [1]. According to official figures, there were a total of 88,387,352 confirmed cases and 1,919,204 deaths registered as of January 12, 2021 [2]. This work was developed in the "12 de Octubre" University Hospital [3], a 1,300-beds tertiary hospital from Madrid Region (Spain), where official sources estimated a total of 434,103 positive cases and 12,001 deaths on January 7, 2021 [4].

In this critical scenario, Electronic Health Records (EHRs) must provide an agile response to the needs of healthcare providers and researchers through a useful data exploitation [5]. To that end, representation and exchange of information with full meaning, known as semantic interoperability [6], is a goal to achieve for primary and secondary uses $[7,8]$. This allows having complete and coherent information regardless of where it was generated, with no errors due to loss of meaning or context [9].

Thus, this work aims to describe how EHRs have been used to supply healthcare and research needs in a major Hospital during the COVID-19 pandemic.

\footnotetext{
${ }^{1}$ Corresponding Author, Miguel Pedrera Jiménez, Hospital Universitario 12 de Octubre, Av. de Córdoba s/n, 28041 Madrid Spain; E-mail: miguel.pedrera@salud.madrid.org.
} 


\section{Methods}

The methodology proposed is supported by previous studies on health information [8, $10]$, and it is formed by two stages. First, new clinical concepts essential to cope with the pandemic situation were identified and standardized. Secondly, these concepts were implemented in Hospital's EHR systems and based on them, data functionalities were provided to healthcare professionals and researchers.

\subsection{Standardization of relevant COVID-19 concepts}

Development of useful data tools require a complete set of structured and standardized variables to be included into the systems and updated in real time. This was carried out gradually as different needs appeared on the daily basis, through collaboration between clinical-domain experts, terminologists and computer scientists. Thus, two subsets of COVID-19 concepts were identified: observations for SARS-COV-2 diagnostic tests and clinical alerts necessary to identify infected and at-risk patients.

\subsubsection{Observable entities for COVID-19 diagnostic tests}

Initially, it was necessary to record data on SARS-COV-2 diagnostic testing in a standardized manner. Two different concepts were identified: detection test by NAA, standardized in March 2020, and rapid immunoassay test, included in September 2020.

Semantic binding was done using LOINC terminology [11], which specified a broad set of concepts for laboratory testing of COVID-19 [12]. Likewise, this standard is part of the recommendations of the Spanish Ministry of Health [13] and has been adopted by the Hospital for the Laboratory Information System (LIS). Table 1 shows this specification of standard observations and the date of implementation.

Table 1. Observable entities for COVID-19 diagnostic tests standardized with LOINC.

\begin{tabular}{ccl}
\hline Concept ID & Implementation date & \multicolumn{1}{c}{ Description } \\
\hline $94315-9$ & $2020-03-17$ & $\begin{array}{l}\text { SARS-related coronavirus E gene [Presence] in Specimen by } \\
\text { NAA with probe detection } \\
\text { SARS-CoV-2 (COVID-19) Ag [Presence] in Respiratory } \\
\text { specimen by Rapid immunoassay }\end{array}$
\end{tabular}

\subsubsection{Clinical alerts and COVID-19 diagnoses}

The most crucial task during pandemic is to classify patients according to whether they are diagnosed, suspected or discarded COVID-19 disease. This identification was done implementing alerts based on WHO specifications [14]. In order to provide a more accurate monitoring of the evolution of patients, additional alerts were included: "respiratory risk" and "patient from nursing home". Thus, since March 2020, every patient admitted to the Hospital has been labelled with them.

Following the Health Ministry of Spain and Hospital strategy [13, 15], these concepts were terminologically linked to SNOMED CT [16], which also has developed several resources for COVID-19 [17]. The latest global version of this terminology was used, as well as the Spanish National Extension [15], for those not found in International Edition. Table 2 shows the set of concepts for COVID-19 alerts standardized with SNOMED-CT and the date of implementation. 
Table 2. Clinical alerts for COVID-19 standardized with SNOMED-CT.

\begin{tabular}{ccl}
\hline Concept ID & Implementation date & \multicolumn{1}{c}{ Description } \\
\hline 63681000122103 & $2020-03-08$ & $\begin{array}{l}\text { Diagnoses disease caused by 2019 novel } \\
\text { coronavirus with specific diagnostic tests } \\
\text { Diagnoses disease caused by 2019 novel } \\
\text { coronavirus without specific diagnostic tests } \\
\text { Suspected disease caused by 2019 novel coronavirus }\end{array}$ \\
82811000122102 & $2020-03-17$ & $\begin{array}{l}\text { Discarded disease caused by 2019 novel coronavirus } \\
\text { At risk of impaired respiratory system function }\end{array}$ \\
688232241000119100 & $2020-03-08$ & Lives in a nursing home \\
704296008 & $2020-04-15$ & \\
\hline
\end{tabular}

\subsection{Implementation in information systems}

Once these concepts were modelled and standardized, they were implemented in Hospital's EHR systems. Our main information system for hospitalization is DXC HCIS version 3.10.5. Thus, different tools were developed in this system to support healthcare of COVID-19 patients. Similarly, research systems were improved to facilitate secondary uses: concepts for COVID-19 were loaded into an i2b2 repository [18], and a mechanism was built for automated population of RedCap databases from EHRs [19].

\section{Results}

The main result of the work was the development of a set of data tools based on the standardized EHRs previously described. These functionalities have two main purposes: improve the management and healthcare and facilitate the research on this new disease.

\subsection{Healthcare tools for COVID-19}

\subsubsection{Clinical alerts}

Clinical alerts to manage the COVID-19 cohort were implemented in the EHR system. The "COVID-19 confirmed case" alert is automatically activated when the system detects a positive diagnostic test. The same way, the "respiratory risk" alert is automatically activated when determined vital signs are abnormal. The rest of alerts must be activated manually by clinicians. Table 3 shows the volume of records stored in the system and how many different patients they refer to, up to January 13, 2021.

Table 3. Volume of clinical alerts from COVID-19 cohort.

\begin{tabular}{lll}
\hline \multicolumn{1}{c}{ Alert } & Records & Patients \\
\hline $\begin{array}{l}\text { Diagnoses disease caused by 2019 novel coronavirus with specific } \\
\text { diagnostic tests }\end{array}$ & 5799 & 5295 \\
$\begin{array}{l}\text { Diagnoses disease caused by 2019 novel coronavirus without specific } \\
\text { diagnostic tests }\end{array}$ & 1242 & 1151 \\
Suspected disease caused by 2019 novel coronavirus & 1722 & 1533 \\
Discarded disease caused by 2019 novel coronavirus & 12057 & 11351 \\
At risk of impaired respiratory system function & 4659 & 4322 \\
Lives in a nursing home & 56 & 56 \\
\hline
\end{tabular}




\subsubsection{Dynamic patient lists}

Based on the standardized alerts described before, dynamic patient lists were implemented. They show, in real time, hospitalized patients that meet criteria established as, for instance, confirmed or diagnosed COVID-19 patients at respiratory risk. In addition, cumulative patient lists were also created for public health purposes.

\subsubsection{Clinical follow-up dashboard}

For the collection and visualization of patient-level data, it was necessary to equip the EHR system with a COVID-19 dashboard. This functionality allows standardized data entry during healthcare activity through clinical forms and displays together data from different systems that, otherwise, would be disaggregated into multiple ones.

\subsection{Research tools for COVID-19}

\subsection{1. i2b2 repository and TriNetX platform}

In order to carry out an adequate secondary use of EHRs [20], known as Real World Data (RWD), the information stored in the healthcare systems is centralized in the Hospital's i2b2 repository [21]. This repository has allowed the Hospital to participate in the 4CE Consortium for COVID-19 of the i2b2 tranSMART Foundation [22]. In the same way, through TriNetX platform [23], our professionals are able to query the contained data to manage the patient cohort and build research hypotheses.

\subsubsection{RedCap databases and ISARIC-WHO CRF}

Data collection is an essential part of clinical research but is very demanding if done manually. To avoid this, in COVID-19's research, a process has been developed to automatically extract, transform, and load EHRs into databases implemented in RedCap, using its API and RStudio [24]. The first database generated in an automated way was the one designed and implemented by the ISARIC Consortium [25].

\section{Conclusions}

The standardization of COVID-19 concepts has allowed the implementation of useful healthcare and management tools based on EHRs. This was essential in a critical situation like the one experienced during the beginning of the pandemic, where the large number of patients and their complexity made it difficult to identify their infection and risk status. Likewise, this has allowed improving clinical research in this new disease through standardized repositories and COVID-19 databases.

\section{Acknowledgment}

This work has been supported by Research Projects PI18/00981 and PI18/01047 funded by Instituto de Salud Carlos III, co-funded by ERDF/ESF. 


\section{References}

[1] Zhu N, Zhang D, Wang W, et al. A Novel Coronavirus from Patients with Pneumonia in China, 2019. N Engl J Med. 2020;382(8):727-733.

[2] WHO COVID-19 $\quad$ Report, 12 January $2021 . \quad$ Available at:https://www.who.int/publications/m/item/weekly-epidemiological-update---12-january-2021.

Accessed March 7, 2021.

[3] "12 de Octubre" Hospital. https://www.comunidad.madrid/hospital/12octubre/. Accessed March 7, 2021.

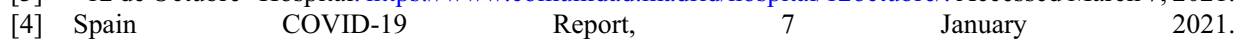
https://www.mscbs.gob.es/profesionales/saludPublica/ccayes/alertasActual/nCov/documentos/Actualiz acion_288_COVID-19.pdf. Accessed March 7, 2021.

[5] Reeves JJ, Hollandsworth HM, Torriani FJ, Taplitz R, Abeles S, Tai-Seale M, Millen M, Clay BJ, Longhurst CA. Rapid response to COVID-19: health informatics support for outbreak management in an academic health system. J Am Med Inform Assoc. 2020 Jun 1;27(6):853-859.

[6] Kalra D, Blobel BG. Semantic interoperability of EHR systems. Stud Health Technol Inform. 2007;127:231-245.

[7] Atreja A, Gordon SM, Pollock DA, Olmsted RN, Brennan PJ; Healthcare Infection Control Practices Advisory Committee. Opportunities and challenges in utilizing electronic health records for infection surveillance, prevention, and control. Am J Infect Control. 2008;36(3):S37-S46.

[8] Pedrera-Jiménez M, García-Barrio N, Cruz-Rojo J, et al. Obtaining EHR-derived datasets for COVID19 research within a short time: a flexible methodology based on Detailed Clinical Models. J Biomed Inform. 2021;115:103697.

[9] Kim MO, Coiera E, Magrabi F. Problems with health information technology and their effects on care delivery and patient outcomes: a systematic review. J Am Med Inform Assoc. 2017;24(2):246-250.

[10] Pedrera M, Serrano P, Terriza A, Cruz J, Varela C, Salamanca A, Garcia N, Salgado C, Sanz C, Hernández L, Alemany M, Muñoz A. Defining a Standardized Information Model for Multi-Source Representation of Breast Cancer Data. Stud Health Technol Inform. 2020 Jun 16;270:1243-1244.

[11] McDonald CJ, Huff SM, Suico JG, Hill G, Leavelle D, Aller R, Forrey A, Mercer K, DeMoor G, Hook J, Williams W, Case J, Maloney P. LOINC, a universal standard for identifying laboratory observations: a 5-year update. Clin Chem. 2003 Apr;49(4):624-33.

[12] LOINC resources for COVID-19. https://loinc.org/sars-coronavirus-2/.Accessed March 7, 2021

[13] Health Ministry of Spain: Minimum data set for clinical reports. Available at: https://www.boe.es/eli/es/rd/2010/09/03/1093. Accessed March 7, 2021.

[14] WHO ICD-10 codes for COVID-19. Available at: https://www.who.int/classifications/icd/COVID-19coding-icd10.pdf. Accessed March 7, 2021.

[15] Health Ministry of Spain: SNOMED CT resources for COVID-19. Available at: https://www.mscbs.gob.es/profesionales/hcdsns/areaRecursosSem/snomed-ct/SNOMED_CT_COVID19.htm. Accessed March 7, 2021.

[16] Donnelly K. SNOMED-CT: The advanced terminology and coding system for eHealth. Stud Health Technol Inform. 2006;121:279-290.

[17] SNOMED CT resources for COVID-19. Available at: https://www.snomed.org/snomed-ct/covid-19. Accessed March 7, 2021.

[18] Murphy SN, Weber G, Mendis M, Gainer V, Chueh HC, Churchill S, Kohane I. Serving the enterprise and beyond with informatics for integrating biology and the bedside (i2b2). J Am Med Inform Assoc. 2010 Mar-Apr;17(2):124-30.

[19] Harris PA, Taylor R, Thielke R, Payne J, Gonzalez N, Conde JG. Research electronic data capture (REDCap)--a metadata-driven methodology and workflow process for providing translational research informatics support. J Biomed Inform. 2009;42(2):377-381.

[20] Kohane IS, Aronow BJ, Avillach P, et al. What Every Reader Should Know About Studies Using Electronic Health Record Data but May be Afraid to Ask J Med Internet Res. 2021 Mar 2;23(3):e22219.

[21] González L, Pérez-Rey D, Alonso E, Hernández G, Serrano P, Pedrera M, Gómez A, De Schepper K, Crepain T, Claerhout B. Building an I2B2-Based Population Repository for Clinical Research. Stud Health Technol Inform. 2020 Jun 16;270:78-82.

[22] Brat GA, Weber GM, Gehlenborg N, et al. International electronic health record-derived COVID-19 clinical course profiles: the 4CE consortium. NPJ Digit Med. 2020 Aug 19;3:109.

[23] TriNetX: COVID-19 Clinical Data. Available at: https://trinetx.com/coronavirus/. Accessed March 7, 2021.

[24] R Project for Statistical Computing. https://www.r-project.org/about.html. Accessed March 7, 2021.

[25] ISARIC-WHO COVID-19 Data Management \& Hosting. https://isaric.org/research/covid-19-clinicalresearch-resources/covid-19-data-management-hosting/. Accessed March 7, 2021. 